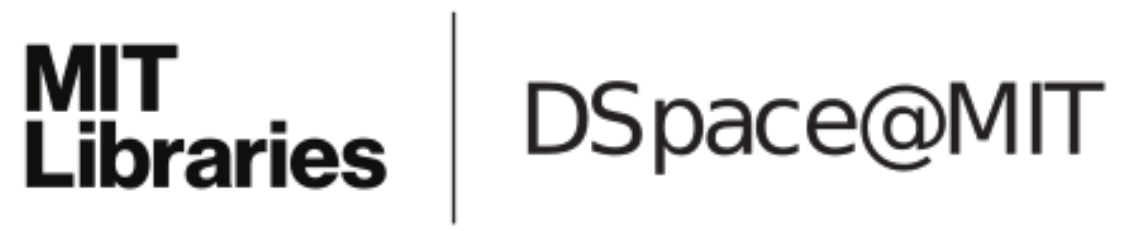

\author{
MIT Open Access Articles
}

Equilibrium analysis for a leader-follower game

with noisy observations: A pace forward in

Witsenhausen's counterexample conjecture

The MIT Faculty has made this article openly available. Please share how this access benefits you. Your story matters.

Citation: Ajorlou, Amir, and Ali Jadbabaie. "Equilibrium Analysis for a Leader-Follower Game with Noisy Observations: A Pace Forward in Witsenhausen's Counterexample Conjecture." 2016 54th Annual Allerton Conference on Communication, Control, and Computing (Allerton), 27-30 September, 2016, Monticello, Illinois, IEEE, 2016.

As Published: http://dx.doi.org/10.1109/ALLERTON.2016.7852232

Publisher: Institute of Electrical and Electronics Engineers (IEEE)

Persistent URL: http://hdl.handle.net/1721.1/113879

Version: Author's final manuscript: final author's manuscript post peer review, without publisher's formatting or copy editing

Terms of use: Creative Commons Attribution-Noncommercial-Share Alike 


\title{
Equilibrium Analysis for a Leader-Follower Game with Noisy Observations: A Pace Forward in Witsenhausen's Counterexample Conjecture
}

\author{
Amir Ajorlou $^{\dagger}$ and Ali Jadbabaie ${ }^{\dagger}$
}

\begin{abstract}
In this paper, we view Witsenhausen's problem as a leader-follower coordination game in which the action of the leader is corrupted by an additive normal noise, before reaching the follower. The leader who observes the realization of the state, chooses an action that minimizes her distance to the state of the world and the ex-ante expected deviation from the follower's action. The follower then makes a noisy observation of the leader's action and chooses an action minimizing her expected deviation from the leader's action. To this end, we analyze the perfect Bayesian equilibria of this game and show that strong complimentarily between the leader and the follower combined with a prior with poor enough precision can give rise to what we call "near piecewise-linear equilibria". As a major consequence, we prove local optimality of a class of slopey quantization strategies which had been suspected of being the optimal solution in the past, based on numerical evidence for Witsenhausen's counterexample.
\end{abstract}

\section{MODEL}

The game consists of a leader $L$ and a follower $F$. Before the agents act, the state of the world $\theta$ is drawn from a normal distribution with zero mean and variance $\sigma^{2}$. The leader can observe the realization of $\theta$ and acts first. The payoff of the leader is given as

$$
u_{L}=-r_{L}\left(\theta-a_{L}\right)^{2}-\left(1-r_{L}\right)\left(a_{F}-a_{L}\right)^{2},
$$

where $a_{F}$ is the action of the follower and $0<r_{L}<1$. The follower makes a private, noisy observation of the leader's action, $s=a_{L}+\delta$ where $\delta \sim N(0,1)$. The payoff of the follower is

$$
u_{F}=-\left(a_{L}-a_{F}\right)^{2}
$$

We consider the perfect Bayesian equilibria of the game and show that they reduce to the Bayes Nash equilibria due to the Gaussian noise in the observation. Denote with $a_{L}^{*}(\theta)$ and $a_{F}^{*}(s)$ the equilibrium strategies, and with $\nu^{*}(\cdot \mid s)$ the follower's belief about leader's action given $s$. Due to the normal noise in the observation, $\nu^{*}(\cdot \mid s)$ is fully determined by $a_{L}^{*}(\theta)$ and the prior as there are no off-equilibrium-path information sets. Equilibrium strategies should thus satisfy

$$
\begin{aligned}
a_{F}^{*}(s)= & \mathbb{E}_{\nu^{*}}\left[a_{L}^{*} \mid s\right]=\int_{-\infty}^{\infty} a_{L} \nu^{*}\left(a_{L} \mid s\right) d a_{L}, \\
a_{L}^{*}(\theta)= & \underset{a_{L}}{\operatorname{argmax}}-r_{L}\left(\theta-a_{L}\right)^{2} \\
& -\left(1-r_{L}\right) \int_{-\infty}^{\infty}\left(a_{F}^{*}(s)-a_{L}\right)^{2} \phi\left(s-a_{L}\right) d s,
\end{aligned}
$$

\footnotetext{
†Institute for Data, Systems, and Society, Massachusetts Institute of Technology (MIT), Cambridge, MA 02139, USA. E-mail: \{ajorlou, jadbabai\}@mit.edu. This work was supported by ARO MURI W911NF-12-1-0509.
}

where $\phi(\cdot)$ denotes the PDF of the standard normal distribution. We can easily characterize the linear equilibria of the game, following [1].

Theorem 1: Linear Bayes strategies of the leader and follower are of the form $a_{F}^{*}(s)=\mu s$ and $a_{L}^{*}(\theta)=\lambda \theta$, where $\mu=\frac{t^{2}}{1+t^{2}}$ and $\lambda=\frac{t}{\sigma}$, and $t$ is a real root of the equation

$$
\frac{r_{L}}{1-r_{L}}(\sigma-t)=\frac{t}{\left(1+t^{2}\right)^{2}}
$$

Our main objective in this paper is to show the existence of an equilibrium with a near piecewise-linear strategy for the leader in the regime where there is strong complementarity ${ }^{1}$ between the leader and the follower (when $r_{L}$ is small) and the prior's precision is poor (or large $\sigma$ ). To this end, and motivated mainly by [1], we focus on regime $\frac{1}{\sigma} \leq r_{L} \sigma^{2} \leq 1$, and aim to show the existence of such an equilibrium for sufficiently large values of $\sigma$ (and hence small $r_{L}$ ).

\section{APPROACH}

Our approach for proving the existence of an equilibrium with a near piecewise-linear strategy for the leader is to identify a set of such strategies for the leader that is invariant under the best response operator.

Given $m \in \mathbb{N}$, consider a partition of the normal distribution $N\left(0, \sigma^{2}\right)$ into $2 m+1$ segments $\cup_{k=-m}^{m} B_{k}^{0}$, with $B_{k}^{0}=\left[b_{k}^{0}, b_{k+1}^{0}\right)$ for $k \in \mathbb{N}_{m}, B_{0}^{0}=\left(b_{-1}^{0}, b_{1}^{0}\right)$, and $B_{-k}^{0}=$ $\left(b_{-k-1}^{0}, b_{-k}^{0}\right]$, with $b_{-k}^{0}=-b_{k}^{0}$ and $b_{m+1}^{0}=-b_{-m-1}^{0}=$ $+\infty$. Denote with $c_{k}^{0}$ the expected value of $\theta \sim N\left(0, \sigma^{2}\right)$ in segment $B_{k}^{0}$, that is, $c_{k}^{0}=\mathbb{E}_{N\left(0, \sigma^{2}\right)}\left[\theta \mid \theta \in B_{k}^{0}\right]$. Clearly, $c_{0}^{0}=0$ and $c_{-k}^{0}=-c_{k}^{0}$ for $k \in \mathbb{N}_{m}$.

We are in particular interested in a partition where the interval endpoints $b_{k}^{0}$ are the midpoints of $\left[c_{k-1}^{0}, c_{k}^{0}\right]$, i.e., $b_{k}^{0}=\frac{c_{k-1}^{0}+c_{k}^{0}}{2}$ for $k \in \mathbb{N}_{m}$. We can show that such a partition exists and is unique. Next, we construct a set of $(2 m+1)$ segmented increasing odd functions, denoted by $A_{L}^{m}\left(r_{L}, \sigma\right)$ satisfying the following properties.

Property 1: For every $a_{L}(\theta) \in A_{L}^{m}\left(r_{L}, \sigma\right)$, there exist $2 m+1$ segments $B_{k}=\left[b_{k}, b_{k+1}\right)$, for $k \in \mathbb{N}_{m}$, $B_{0}=\left(-b_{1}, b_{1}\right)$, and $B_{-k}=\left(b_{-k-1}, b_{-k}\right]$, with $b_{m+1}=$ $-b_{-m-1}=+\infty$ such that:

- $a_{L}(\theta)$ is increasing and odd (i.e., $a_{L}(-\theta)=-a_{L}(\theta)$ ), and is smooth over each interval.

- $a_{L}(\theta)$ has a unique fixed point in each segment. That is, for each interval $B_{k},(-m \leq k \leq m)$, there exists a unique $c_{k} \in B_{k}$ such that $a_{L}\left(c_{k}\right)=c_{k}$, with $c_{0}=0$.

\footnotetext{
${ }^{1}$ Games of strategic complementarities are those in which the best response of each player is increasing in actions of others.
} 
We also impose a constraint on the slope of $a_{L}(\theta)$ in each interval, keeping the slope very close to $r_{L}$, as well as a linear bound on $a_{L}(\theta)$ in the tail. More precisely, we impose the following property:

Property 2: For every $-m<k<m$ and $\theta \in B_{k}, \underline{r} \leq$ $\frac{d}{d \theta} a_{L}(\theta) \leq \bar{r}$, where $\underline{r}=r_{L}\left(1-0.5 r_{L}^{2} \sigma^{2}\right)$ and $\bar{r}=r_{L}(1+$ $\left.2.5 r_{L}^{2} \sigma^{2}\right)$. For the tail interval $B_{m}, \underline{r} \leq \frac{d}{d \theta} a_{L}(\theta) \leq \bar{r}$ for $b_{m}<\theta<c_{m}+\sigma^{2}$. For $\theta>c_{m}+\sigma^{2}$ we have $a_{L}(\theta) \leq$ $c_{m}+5 r_{L}\left(\theta-c_{m}+\sigma\right) .^{2}$

The larger $\sigma$, the closer $\bar{r}$ and $\underline{r}$ to $r_{L}$. For instance, choosing $\sigma \geq 16$ ensures $\bar{r}<1.01 r_{L}$ and $\underline{r}>0.998 r_{L}$. Finally, we impose the constraint that interval endpoints $b_{k}$ remain close to midpoints of $\left[c_{k-1}, c_{k}\right]$ and that fixed points $c_{k}$ remain close to $c_{k}^{0}$ 's.

Property 3: For every $k \in \mathbb{N}_{m}$,

$$
\left|b_{k}-\frac{c_{k-1}+c_{k}}{2}\right| \leq 0.1 r_{L} \sigma
$$

Moreover,

$$
\left|c_{k}-c_{k}^{0}\right| \leq \frac{2 k(2 m-k) \zeta}{r_{L}},
$$

where $\zeta=0.44 r_{L}^{2} \sigma^{2}+(2 m+3.4) r_{L}^{2} \sigma$.

Our proof strategy is then to show that for any $m \in \mathbb{N}$, there exists $\sigma_{m}>0$ such that, for every $\sigma \geq \sigma_{m}$ in regime $\frac{1}{\sigma} \leq r_{L} \sigma^{2} \leq 1$, the set of strategies $A_{L}^{m}\left(r_{L}, \bar{\sigma}\right)$ characterized by Property 1-3 is invariant under the best response operator. ${ }^{3}$

\section{MAIN RESUlTS}

We summarize the main result of the paper in the theorem below.

Theorem 2: Consider the regime $\frac{1}{\sigma} \leq r_{L} \sigma^{2} \leq 1$ and a given $m \in \mathbb{N}$. Then, there exists $\sigma_{m}>0$ such that for any $\sigma \geq \sigma_{m}$, the set of $(2 m+1)$-segmented strategies $A_{L}^{m}\left(r_{L}, \sigma\right)$ for the leader, characterized by Property 1-3, is invariant under the best response. Moreover, the game described in Section I has an equilibrium for which $a_{L}^{*}\left(\theta, r_{L}, \sigma\right) \in$ $A_{L}^{m}\left(r_{L}, \sigma\right)$.

Given $m \in \mathbb{N}$, the maximum deviation of fixed points $c_{k}$ from their counterparts $c_{k}^{0}$ for strategies in $A_{L}^{m}\left(r_{L}, \sigma\right)$ is upper-bounded by $2 m^{2}\left(0.44 r_{L} \sigma^{2}+(2 m+3.4) r_{L} \sigma\right)$, according to (6). This upper bound does not grow unboundedly with $\sigma$ in the regime $\frac{1}{\sigma} \leq r_{L} \sigma^{2} \leq 1$. Furthermore, if $\sigma \rightarrow+\infty$ along a path where $r_{L} \sigma^{2} \rightarrow 0$, this upper bound approaches zero, hence asymptotically identifying the equilibrium strategy.

Proposition 1: Let $a_{L}^{0}\left(\theta, r_{L}, \sigma\right)$ be the $(2 \mathrm{~m}+1)$-segmented piecewise-linear strategy with $B_{k}^{0}$ 's as segments and $c_{k}^{0}$ 's as fixed points and fixed common slope $r_{L}$, that is, $a_{L}^{0}\left(\theta, r_{L}, \sigma\right)=c_{k}^{0}+r_{L}\left(\theta-c_{k}^{0}\right)$ for $\theta \in B_{k}^{0}$. Then,

$$
\lim _{\substack{r_{L} \sigma^{2} \rightarrow 0 \\ \sigma \rightarrow+\infty}} \mathbb{E}_{N\left(0, \sigma^{2}\right)}\left[\left|a_{L}^{*}\left(\theta, r_{L}, \sigma\right)-a_{L}^{0}\left(\theta, r_{L}, \sigma\right)\right|\right] \rightarrow 0 .
$$

In [1], Witsenhausen proves the existence of a nonlinear controller which outperforms the optimal linear strategy for

\footnotetext{
${ }^{2}$ We only state the properties only for $\theta \geq 0$. The counterpart for $\theta \leq 0$ is immediate since the function is odd.

${ }^{3}$ See the full version of this paper in [2] for the steps required to verify the invariance of $A_{L}^{m}\left(r_{L}, \sigma\right)$.
}

a simple two-stage LQG decentralized control problem. The equivalent regime under our setup is $k^{2} \sigma^{2}=1$, where $k^{2}=\frac{r_{L}}{1-r_{L}}$. Witsenhausen shows that for large enough values of $\sigma$ the optimal controller in this regime is nonlinear. It has been a long-standing conjecture that the optimal controller is a near piecewise-linear controller. Theorem 2 proves the existence of an equilibrium for the game of Section I with a near piecewise-linear strategy for the leader for sufficiently large $\sigma$ in the regime $\frac{1}{\sigma} \leq r_{L} \sigma^{2} \leq 1$; This implies the existence of a slopey quantized local optimum 4 for Witsenhausen's counterexample in this regime (it is easy to verify that $k^{2} \sigma^{2}=1$ falls in this regime, since $k^{2} \sigma^{2}=1$ yields $\frac{1}{\sigma}<r_{L} \sigma^{2}=1-r_{L}<1$ for $\sigma>2$ ). Below, we illustrate Theorem 2 for the special cases of $m=1,2$ by specifying explicit values for $\sigma_{m}$ which guarantee the existence of 3-segmented and 5-segmented near piecewiselinear equilibria, respectively.

Proposition 2 (3-Segmented Equilibria): Suppose that $\frac{1}{\sigma} \leq r_{L} \sigma^{2} \leq 1$ and $\sigma \geq 16$. Then, the game described in Section I has an equilibrium with $a_{L}^{*}\left(\theta, r_{L}, \sigma\right) \in A_{L}^{1}\left(r_{L}, \sigma\right)$; $a_{L}^{*}\left(\theta, r_{L}, \sigma\right)$ is a 3 -segmented near piecewise-linear strategy possessing Property 1-3.

It is to be noted that smaller values for $\sigma_{m}$ may work here, and similarly in Proposition 3. These values are obtained using the bounds derived for generic $m$. Of course, given a specific value of $m$, these bounds can be tightened much further which may result in smaller values for $\sigma_{m}$.

Proposition 3 (5-Segmented Equilibria): Suppose that $\frac{1}{\sigma} \leq r_{L} \sigma^{2} \leq 1$ and $\sigma \geq 60$. Then, the game described in Section I has an equilibrium with $a_{L}^{*}\left(\theta, r_{L}, \sigma\right) \in A_{L}^{2}\left(r_{L}, \sigma\right)$; $a_{L}^{*}\left(\theta, r_{L}, \sigma\right)$ is a 5-segmented near piecewise-linear strategy possessing Property 1-3.

\section{Conclusions}

We viewed Witsenhausen's counterexample as a leaderfollower game of incomplete information. We showed the existence of nonlinear perfect Bayesian equilibria in the regime where there is strong complementarity between the leader and the follower when the prior has very poor precision. Our results provide the first analytical proof for the local optimality of near piecewise-linear controllers for the Witsenhausen's counterexample, where the optimal controller is conjectured to be a slopey quantizer.

\section{REFERENCES}

[1] H. S. Witsenhausen, "A counterexample in stochastic optimum control," SIAM J. Control, vol. 6, pp. 131-147, 1968.

[2] A. Ajorlou and A. Jadbabaie, "Slopey quantizers are locally optimal for witsenhausen's counterexample," available at: http://arxiv. org/abs/1604.03806, 2016.

\footnotetext{
${ }^{4}$ This is indeed stronger than a local optimum since, at an equilibrium, fixing one player's strategy the deviation in the other player's strategy does not have to be local.
} 